\title{
Sequence-based typing of Legionella pneumophila serogroup 1 clinical isolates from Belgium between 2000 and 2010
}

E Vekens (labomicro@uzbrussel.be) ${ }^{1}, 0$ Soetens ${ }^{1}, \mathrm{R}$ De Mendonça² ${ }^{2}$, F Echahidi $^{1}$, S Roisin $^{2}$, A Deplano $^{2}$, L Eeckhout ${ }^{1}$,

W Achtergael ${ }^{1}$, D Piérard ${ }^{1}, 0$ Denis ${ }^{2}$, I Wybo ${ }^{1}$

1. National Reference Centre for Legionella pneumophila, Department of Microbiology and Infection Control, Universitair Ziekenhuis Brussel, Vrije Universiteit Brussel (VUB), Brussels, Belgium

2. National Reference Centre for Legionella pneumophila, Department of Microbiology, Erasme Hospital, Université Libre de Bruxelles, Brussels, Belgium

Vekens E, Soetens O, De Mendonça R, Echahidi F, Roisin S, Deplano A, Eeckhout L, Achtergael W, Piérard D, Denis O, Wybo I. Sequence-based typing of

Legionella pneumophila serogroup 1 clinical isolates from Belgium between 2000 and 2010. Euro Surveill. 2012;17(43):pii=20302. Available online: http://www. eurosurveillance.org/ViewArticle.aspx?Articleld $=20302$

Article submitted on 07 May 2012 / published on 25 October 2012

Sequence-based typing (SBT) is a discriminatory method widely used to genotype Legionella pneumophila strains. A total of 86 clinical L. pneumophila serogroup 1 (sg1) isolates, collected between January 2000 and December 2010 in the two Belgian National Reference Centres for Legionella pneumophila, were genotyped using the internationally standardised SBT protocol of the European Working Group for Legionella Infections (EWGLI). The isolates could be classified into 31 different sequence types (ST, index of diversity: 0.879). The obtained STs were submitted to the EWGLI SBT-database for L. pneumophila. In our study, ST47 (27.9\%) and $\mathrm{ST}_{1}(19.8 \%)$ were the most frequently detected STs. The detected profiles were a combination of both frequently isolated and unique STs, and of both worldwide distributed and more local strains. Two STs, ST880 and ST881, were new to the EWGLI database. In conclusion, we characterised L. pneumophila sg1 isolates with the SBT method, and created a Belgian profile database that will be useful for future epidemiological studies.

\section{Introduction}

Legionella spp. are rod-shaped, gram-negative bacteria, which are ubiquitously spread in aqueous environments [1] where they survive as intracellular parasites of protozoa [2]. Legionella is transmitted to humans by inhalation of contaminated aerosols. Common sources are air conditioning systems, cooling towers, dental devices and showerheads [3]. Legionellosis can appear in two distinct clinical presentations, Legionnaires' disease (LD), a mild to fatal pneumonia with an approximate case fatality rate of $6.6 \%$ [4], and Pontiac fever, an acute self-limited influenza-like illness [5]. One species of Legionella, L. pneumophila, is the aetiological agent of approximately $90 \%$ of legionellosis cases, and serogroup 1 (sg 1) accounts for about $84 \%$ of these cases [6]. Notification of legionellosis to the health authority is mandatory in Belgium. Most reported cases are single infections, but outbreaks do occur [7].

The characterisation of clinical isolates by molecular typing methods is essential for epidemiological investigations of sporadic cases and outbreaks. L. pneumophila sg1 isolates can be genotyped by sequence-based typing (SBT) using the seven loci (flaA, pilE, asd, mip, mompS, proA and neuA) proposed by the European Working Group on Legionella Infections (EWGLI, renamed to European Study Group for Legionella Infections, ESGLI, since September 2012) $[8,9]$. We determined the SBT of clinical L. pneumophila sg1 isolates recovered over a 10-year period in Belgium, and compared these results to available data in other countries.

\section{Methods}

\section{Legionella pneumophila isolates}

Clinical isolates of $L$. pneumophila sg1 collected between January 2000 and December 2010 in the laboratories of Microbiology of UZ Brussel and ULBErasme, the two Belgian National Reference Centres for L. pneumophila, were retrospectively analysed. Clinical laboratories of both general and university hospitals may refer clinical samples for culture and PCR or strains for molecular typing to the reference centres. This service is free of charge for all clinical laboratories and supported by the health authorities. During the 10-year study period, we gathered 106 L. pneumophila sg 1 isolates from 29 hospitals, of which 91 were available for further SBT analysis. All isolates were unduplicated and collected from different patients with LD (diagnosed with pneumonia according to the EU case definition [10]). Epidemiological data for each isolate included the patient's age and sex, hospital from which specimens were submitted, and if available, the patient's place of residence and probable origin 
of infection. A case of LD was considered nosocomial if the patient had been hospitalised during the entire incubation period (10 days), or travel-associated if the patient had spent at least one night away from home, either in Belgium or abroad, 10 days before onset of the symptoms. For the other cases, the patient's place of residence, if available, was used as a proxy for the place of infection. Clinical cases were subdivided into related or single cases. Isolates were considered related to each other if they were recovered, within the same year, from patients with a probable or confirmed common source of contamination.

\section{Identification methods}

The L. pneumophila isolates were cultured on buffered charcoal yeast extract agar (Legionella CYE, Oxoid, UK) supplemented with ACES buffer, alpha-ketoglutarate, ferric pyrophosphate and L-cysteine and with and without the antibiotics cefamandole, polymyxin $\mathrm{B}$ and anisomycin (Legionella BMPA-alpha selective supplement SRo111B, Oxoid, UK). Isolates were identified as L. pneumophila by determination of cellular fatty acid composition by gas-chromatography $[11,12]$. Identification to serogroup level was performed by latex agglutination using Microgen Legionella latex kit (Microgen Bioproducts Ltd., UK) or Oxoid Legionella latex test (Oxoid, UK). Isolates were stored at $-80^{\circ} \mathrm{C}$ in nutrient broth supplemented with $15 \%$ glycerol until analysis.

\section{Molecular typing}

The first recovered isolate from each group of related isolates and all unrelated isolates were included and genotyped using the standard protocol from the EWGLI SBT scheme $[8,9,13]$. DNA was prepared directly from colonies by incubating the bacterial suspensions in $250 \mu \mathrm{L}$ Tris-EDTA buffer for molecular biology $(\mathrm{pH} 8$, Sigma Aldrich, Bornem, Belgium) at $100^{\circ} \mathrm{C}$ for $10 \mathrm{~min}-$ utes. Polymerase chain reaction (PCR) was performed using primers targeting the gene loci flaA [13], pilE [8], asd [8], mip [8], mompS [8,13], proA [8] and neuA [9] on an iCycler (Bio-Rad). Purification of PCR products and sequencing with the amplification primers, except for the reverse sequencing primer of mompS [8], was done by VIB Genetic Service Facility (University of Antwerp, Wilrijk, Belgium). Sequence alignment, trimming and allele designation were performed using the online EWGLI Sequence Quality Tool. The obtained STs were submitted to the EWGLI SBT-database (http://www. hpa-bioinformatics.org.uk/legionella/legionella_sbt/ php/sbt_homepage.php). The data from our study were compared to literature and to data submitted to the EWGLI database until 25 January 2012.

\section{Statistical analysis}

The index of diversity (IOD) was calculated by using Hunter and Gaston's modification of Simpson's index of diversity as previously described [14]. The MannWhitney U-test was used to assess whether there was a significant change in frequency of ST isolation over years. Statistics were performed with Analyse-it for
Microsoft Excel (version 2.21 Analyse-it Software Ltd, UK). $\mathrm{P}$ values $(0.050$ were considered as statistically significant.

\section{Results}

\section{Clinical isolates}

From 2000 to 2010, 212 cases of LD were confirmed by the Belgian National Reference Laboratories. Of these, $122(57.5 \%)$ were diagnosed by culture and isolation of Legionella spp. L. pneumophila sg1 was the causative agent in the majority of culture-confirmed cases $(n=106,86.9 \%)$. Of the 91 L. pneumophila serogroup 1 clinical isolates available for SBT analysis, 82 were from clinically single cases. Over the study period, four outbreaks occurred (involving nine cases in total) and all of them were linked to a hospital facility. After inclusion of the first isolate for each outbreak (no discrepancy was observed between isolates from the same outbreak), 86 isolates were included in our study and typed by SBT analysis.

\section{Epidemiological data}

The median age of included patients was 58 years (range: 19-92 years; excluding one patient whose age was unknown) and 62 patients (73\%) were men. There were 15 travel-associated cases (17.4\%), eight nosocomial cases (9.3\%), 11 community-acquired cases $(12.8 \%)$ and 52 cases for which the source could not be identified (60.5\%) (Table).

Legionella pneumophila sequence-based typing SBT analysis assigned the 86 unrelated clinical isolates to 31 distinct STs (IOD: 0.879). As shown in the Table, $47.7 \%$ of the isolates belonged to two main STs with 24 and 17 isolates, respectively. Eight STs consisted of groups containing between two and five isolates and the remaining STs $(n=21)$ accounted for only one single isolate each.

The ST with the largest number of isolates $(n=24)$ was ST 47 , which represented $27.9 \%$ of all clinical isolates. The ST47 profile was found ubiquitously across Belgium as shown in Figure 1. Two ST47 isolates were associated with travelling to France and Italy, respectively. A suspected source could be identified for a further three cases: a decorative fountain, a visit to a garden centre and the maintenance of an outdoor swimming pool.

Genotype ST1 was found in 17 isolates (19.8\%). The geographic distribution of $\mathrm{ST}_{1}$ in Belgium was mainly restricted to Brussels (Figure 1). Six isolates were associated with nosocomial infections. Four of these isolates were found between 2000 and 2005 in one hospital, two of which were related to outbreaks involving two cases each. One ST1 isolate was travelassociated; the infection was acquired in Spain.

Other major STs were ST6 $(n=5), S_{23}(n=5), S_{42}(n=3)$ and ST62 $(n=3)$. One of the ST6 isolates was associated with a nosocomial outbreak involving three cases. 
Distribution of Legionella pneumophila serogroup 1 isolates by sequence type and origin of acquisition, Belgium, January 2000-December $2010(n=86)$

\begin{tabular}{|c|c|c|c|c|c|c|c|}
\hline ST & Allelic profile ${ }^{a}$ & $\mathrm{~N}$ & $\%$ & Community-acquired & Travel-associated & Nosocomial & Undetermined origin \\
\hline $\mathrm{ST} 47$ & $5,10,22,15,6,2,6$ & 24 & 27.9 & 6 & 2 & 0 & 16 \\
\hline $\mathrm{ST}_{1}$ & $1,4,3,1,1,1,1$ & 17 & 19.8 & 1 & 1 & 6 & 9 \\
\hline ST6 & $1,4,3,1,1,1,15$ & 5 & 5.8 & 0 & 0 & 1 & 4 \\
\hline $\mathrm{ST} 23$ & $2,3,9,10,2,1,6$ & 5 & 5.8 & 0 & 1 & 0 & 4 \\
\hline $\mathrm{ST}_{42}$ & $4,7,11,3,11,12,9$ & 3 & 3.5 & 1 & 2 & 0 & 0 \\
\hline ST62 & $8,103,15,18,1,6$ & 3 & 3.5 & 0 & 1 & 0 & 2 \\
\hline ST9 & $3,10,1,3,14,9,11$ & 2 & 2.3 & 0 & 0 & 0 & 2 \\
\hline $\mathrm{ST} 48$ & $5,2,22,27,6,10,12$ & 2 & $2 ., 3$ & 0 & 0 & 0 & 2 \\
\hline ST110 & $2,10,3,3,9,4,9$ & 2 & 2.3 & 1 & 0 & 1 & 0 \\
\hline ST664 & $3,13,1,3,14,9,9$ & 2 & 2.3 & 0 & 1 & 0 & 1 \\
\hline ST109 & $5,1,22,15,6,10,6$ & 1 & 1.2 & 0 & 0 & 0 & 1 \\
\hline $\mathrm{ST} 146$ & $2,10,18,10,2,1,6$ & 1 & 1.2 & 0 & 0 & 0 & 1 \\
\hline $\mathrm{ST} 16$ & $2,10,18,10,2,1,9$ & 1 & 1.2 & 0 & 0 & 0 & 1 \\
\hline ST196 & $3,10,1,28,14,9,11$ & 1 & 1.2 & 0 & 0 & 0 & 1 \\
\hline $\mathrm{ST} 20$ & $2,3,18,15,2,1,6$ & 1 & 1.2 & 0 & 1 & 0 & 0 \\
\hline $\mathrm{ST} 22$ & $2,3,6,10,2,1,6$ & 1 & 1.2 & 0 & 0 & 0 & 1 \\
\hline $\mathrm{ST} 301$ & $2,10,3,12,9,4,9$ & 1 & 1.2 & 1 & 0 & 0 & 0 \\
\hline $\mathrm{ST} 345$ & $6,10,19,3,19,4,11$ & 1 & 1.2 & 0 & 0 & 0 & 1 \\
\hline $\mathrm{ST} 37$ & $3,4,1,1,14,9,11$ & 1 & 1.2 & 0 & 0 & 0 & 1 \\
\hline $\mathrm{ST} 438$ & $3,10,1,1,14,9,15$ & 1 & 1.2 & 0 & 0 & 0 & 1 \\
\hline ST479 & $5,1,22,10,6,10,12$ & 1 & 1.2 & 1 & 0 & 0 & 0 \\
\hline $\mathrm{ST} 487$ & $3,6,1,28,14,11,11$ & 1 & 1.2 & 0 & 1 & 0 & 0 \\
\hline ST579 & $3,13,1,3,14,9,11$ & 1 & 1.2 & 0 & 1 & 0 & 0 \\
\hline ST744 & $2,3,5,5,18,1,10$ & 1 & 1.2 & 0 & 0 & 0 & 1 \\
\hline ST751 & $8,10,3,15,9,14,6$ & 1 & 1.2 & 0 & 1 & 0 & 0 \\
\hline ST77 & $6,10,14,10,2,3,6$ & 1 & 1.2 & 0 & 1 & 0 & 0 \\
\hline ST862 & $4,17,11,15,29,12,20$ & 1 & 1.2 & 0 & 1 & 0 & 0 \\
\hline ST880 & $6,10,19,3,2,4,11$ & 1 & 1.2 & 0 & 0 & 0 & 1 \\
\hline ST881 & $7,7,17,3,13,11,9$ & 1 & 1.2 & 0 & 1 & 0 & 0 \\
\hline ST94 & $12,8,11,5,20,12,2$ & 1 & 1.2 & 0 & 0 & 0 & 1 \\
\hline ST99 & $4,8,11,5,29,12,10$ & 1 & 1.2 & 0 & 0 & 0 & 1 \\
\hline \multirow[t]{2}{*}{ Sum } & & 86 & & 11 & 15 & 8 & 52 \\
\hline & & & & $(12.8 \%)$ & (17.4\%) & (9.3\%) & $(60.5 \%)$ \\
\hline
\end{tabular}

ST: sequence type

a Sequence of genes flaA, pilE, asd, mip, mompS, proA, neuA.

ST110 was recovered twice among our isolates: in one nosocomial and one community-acquired infection. These two clinical isolates could be matched with environmental isolates recovered from shower water in a hospital and from water in a service flat, respectively. The nosocomial isolate was associated with a hospitaloutbreak involving two cases.

Two STs were new to the EWGLI database: ST881 was detected in an isolate from a patient probably infected in Spain, and ST880 was detected in a clinical isolate of unknown origin.

Sequence type distribution by year of isolation The profile distribution of $L$. pneumophila sg1 clinical isolates by year of isolation was heterogeneous (Figure 2). From 2000 through $2005, \mathrm{ST}_{1}$ was regularly isolated and accounted yearly for $14.3 \%$ to $60 \%$ of clinical isolates. The incidence of ST1 was significantly lower from 2006 through 2010 with an average recovery in $8.2 \%$ of clinical isolates $(p=0.009)$. Although isolation of ST47 
Geographic distribution of Legionella pneumophila serogroup 1 isolates with sequence type ST1 and ST47 in Belgium (A) and in Brussels (B), January 2000-December $2010(n=33)$

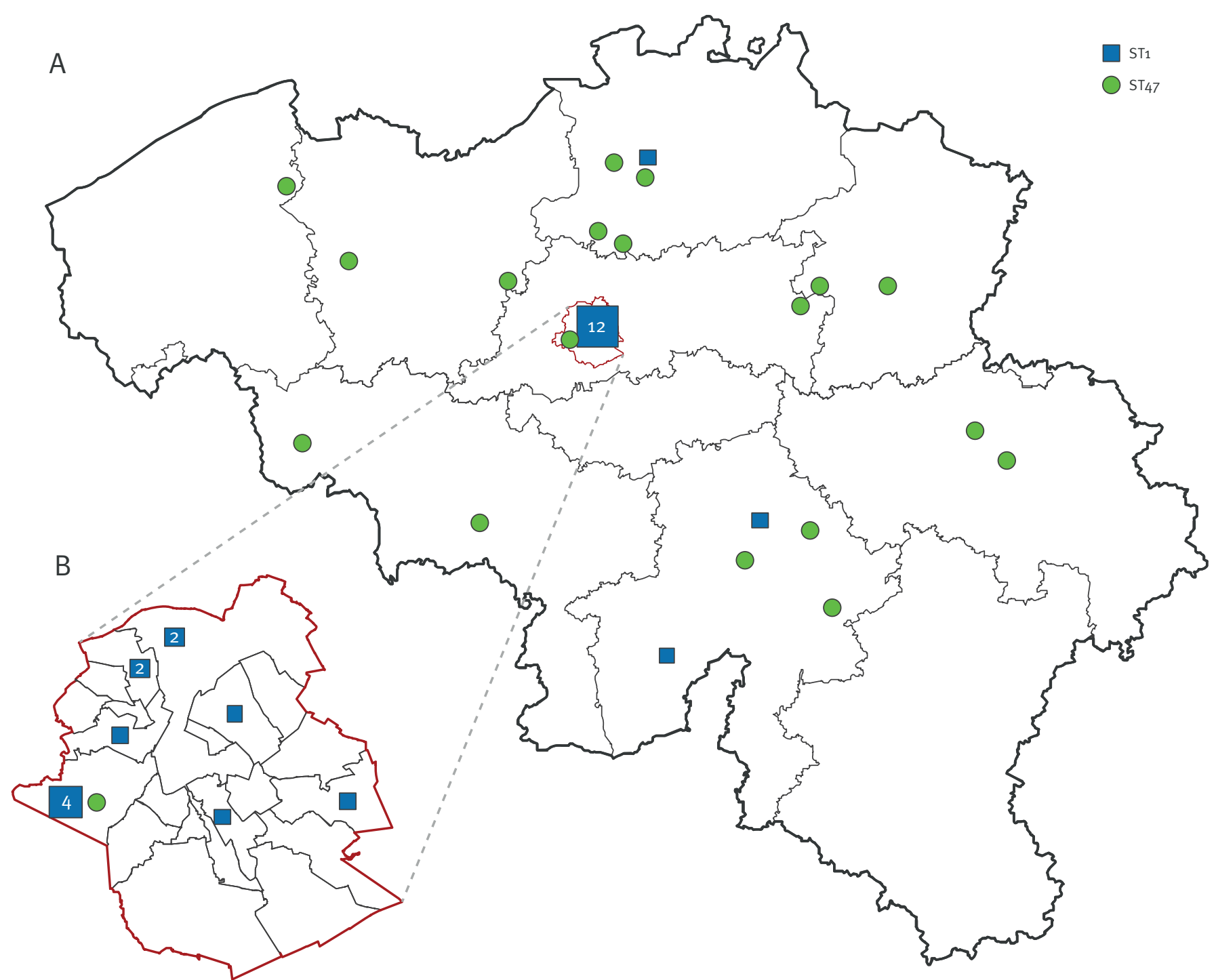

The number of cases is shown for locations with more than one case. Travel-associated cases $(n=3)$ and cases with no available geographic information $(n=5)$ are not shown.

peaked in 2007 ( $80 \%$ of clinical isolates), there was no significant difference in the frequency of ST47 isolation between 2000-05 and 2006-10 $(p=0.662)$.

\section{Discussion}

This report represents the first SBT analysis of L. pneumophila serogroup 1 clinical isolates in Belgium from 2000 to 2010. Over this period, 212 cases of LD were confirmed by the Belgian National Reference Laboratories, of which 122 (57.5\%) were diagnosed by the isolation of Legionella. In line with other reports, the majority of culture-confirmed LD cases in Belgium was caused by L. pneumophila sg1. The incidence of other serogroups (13 clinical isolates: four sg6, three sg4, one sg10, one sg3, three sg2-15 and one undefined) and non-pneumophila species of Legionella (two L. bozemanii and one L. longbeachae) (data not shown) was similar to the distribution in the rest of Europe $[4,15]$.

Diagnosis of LD in Belgium is based on culture, PCR, serology and urinary antigen detection. Since many clinical laboratories in Belgium use the urinary antigen test as a primary diagnosis tool, culture and strain isolation of $L$. pneumophila from respiratory samples is, in our experience, seldom undertaken in less severe cases. In the future, clinicians should be encouraged to refer respiratory samples for Legionella culture in case of confirmed LD. During the 10-year study period, we gathered 106 L. pneumophila serogroup 1 isolates, of which 91 were available for further SBT analysis. The incidence of Legionnaires' disease in Belgium between 2003 and 2010 was about 138 cases per year $[4,16,17,18,19]$. Therefore, our study may not represent 
all LD cases, but it gives a good representation of the circulating isolates. A comparison of epidemiological characteristics of included cases and non-culture verified cases showed no statistically significant difference in age, sex and distribution into nosocomial and travel-associated cases (data not shown). Although the cases for which urine samples were referred to our laboratories could be biased, this finding suggests that the included cases might be a good representation of all cases of $L$. pneumophila sg1 infection in Belgium.

In our study, 31 distinct allelic STs were detected among 86 clinical L. pneumophila sg1 isolates which reflects a high profile diversity. The index of diversity (0.879) in our investigation was lower than that described previously in Japan (0.979) [20], the United States (0.946) [21], Canada (0.964) [22] and England and Wales (0.901) [23]. This could be explained by the smaller geographic area of Belgium as compared to the other countries. The combination of SBT analysis with monoclonal antibody subgrouping [24] would allow a further characterisation of the clinical isolates. This subgrouping can have relevance for source investigation especially for common STs, like ST1 [22,25].

The detected STs in our study are a combination of both frequently isolated and unique STs, and of both worldwide distributed and more local STs. The most common ST in Belgium was ST47 (27.9\%). This observation is in accordance with published data; ST47 is also the major ST found in France, the Netherlands, and England and Wales [23,26,27]. The most frequent ST reported in the world, ST1, was found in 17 isolates (19.8\%). ST6 was recovered from five isolates and is an important but more local strain since isolation of this genotype is restricted to the region of Germany, France and Belgium according to the data submitted to the EWGLI database. Strains occurring worldwide that were responsible for three to five cases in Belgium included $\mathrm{ST}_{23}$ and ST42. The ST23 genotype has repeatedly been isolated from clinical cases in Europe, mostly in France $(n=291)$ and the Netherlands $(n=27)$, according to the EWGLI database. Interestingly, one of our cases was associated with travelling to France. Apart from the frequent isolation in Europe [25,28], this ST was also found to be responsible for two large outbreaks in Japan associated with a bath facility [20]. The ST42 profile is also widely distributed and most frequently isolated in the Netherlands $(n=30)$, France $(n=17)$ and England ( $n=16)$ according to the EWGLI database. Data from England and Wales showed that this profile was often associated with travelling outside the UK. This is in accordance with our data since two of our three ST42 strains were associated with travel to Turkey and Italy. Only one strain with ST37 profile was detected among our isolates. In contrast, this profile accounted for $11.4 \%$ of clinical isolates in England and Wales [23] and was detected in 21 clinical isolates in Canada [29].

During this 10-year surveillance period, only four small nosocomial outbreaks were detected. This is in contrast
FIGURE 2

Distribution of Legionella pneumophila serogroup 1 sequence types by year of isolation, Belgium,

January 2000-December $2010(n=86)$

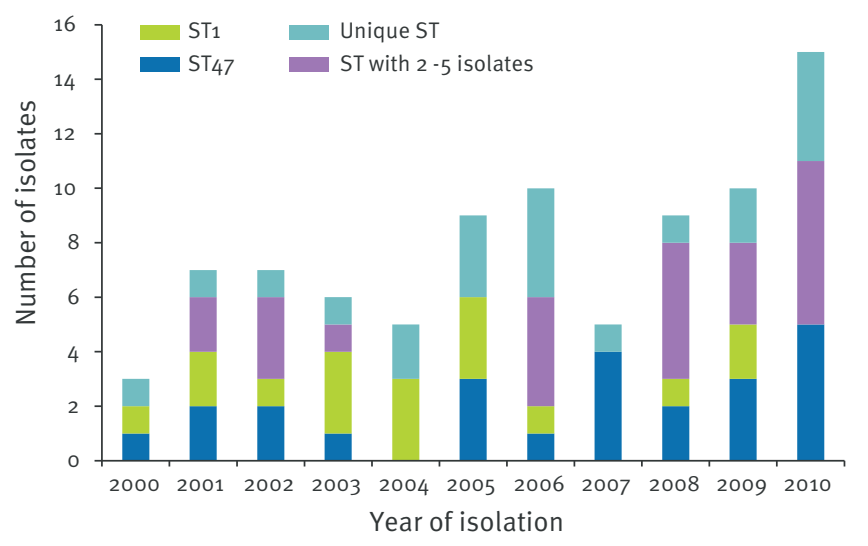

ST: sequence type.

with the large ST36 outbreak of 1999 at Kappellen in Belgium, where more than 90 cases occurred during a fair [7]. Of notice is that the ST36 from the 1999 outbreak was not detected in the present study. Of the four outbreaks recorded in Belgium during the study period, two were associated with $\mathrm{ST} 1$, while the two other outbreaks were associated with ST6 or ST110, respectively. Four of the $\mathrm{ST}_{1}$ nosocomial infections occurred within the same hospital. This institution was probably colonised by $\mathrm{ST}_{1}$, which caused two outbreaks and two sporadic cases over several years. In France and Canada, this sequence type was found to be responsible for sporadic as well as outbreak cases $[22,28,29]$.

In the 10-year study period, differences could be observed in profile distribution by year of isolation. The decreased incidence of ST1 over the years is in accordance with observations made in Canada and Japan $[20,29]$. Irrespective of the temporal differences between the ST47 and ST1 strains, the pattern of geographical distribution varies between both strains. The ST47 profile showed a dispersed distribution in Belgium whereas detection of the ST1 profile was generally restricted to Brussels.

As demonstrated in our study and in previous publications, Legionnaires' disease occurs both in sporadic and epidemic forms. Prompt recognition of LD cases and outbreaks is necessary to control epidemics quickly and to treat patients effectively. As a result of this study a Belgian national database of $L$. pneumophila SBT profiles was created, which is a useful tool for the investigation and management of local outbreaks. Our data were uploaded to the EWGLI SBT-database which allows comparison between countries and is valuable in epidemiological investigations, since cases might be dispersed over different regions and countries. 


\section{References}

1. Fliermans CB, Cherry WB, Orrison LH, Smith SJ, Tison DL, Hope $\mathrm{DH}$. Ecological Distribution of Legionella pneumophila. Appl Environ Microbiol. 1981;41(1):9-16.

2. Fields BS, Benson RF, Besser RE. Legionella and Legionnaires' Disease: 25 Years of Investigation. Clin Microbiol Rev. 2002;15(3):506-26.

3. Steinert M, Hentschel U, Hacker J. Legionella pneumophila: an aquatic microbe goes astray. FEMS Microbiology Rev. 2002;26:149-62.

4. Joseph CA, Ricketts KD, on behalf of the European Working Group for Legionella Infections. Legionnaires' disease in Europe 2007 - 2008. Euro Surveill. 2010;15(8):pii=19493. Available from: http://www.eurosurveillance.org/ViewArticle. aspx?Articleld=19493

5. Glick TH, Gregg MB, Berman B, Mallison G, Rhodes WWJr, Kassanoff I. Pontiac fever. An epidemic of unknown etiology in a health department: I. Clinical and epidemiological aspects. Am J Epidemiol. 1978;107(2):149-60.

6. Yu VL, Plouffe JF, Pastoris MC, Stout JE, Schousboe M, Widmer $A$, et al. Distribution of Legionella species and serogroups isolated by culture in patients with sporadic communityacquired legionellosis: an international collaborative survey. J Infect Dis. 2002;186(1):127-8.

7. De Schrijver K, Van Bouwel E, Mortelmans L, Van Rossom P, De Beukelaer T, Vael C, et al. An outbreak of Legionnaire's disease among visitors to a fair in Belgium, 1999. Euro Surveill. 2000;5(11):pii=7. Available from: http://www.eurosurveillance. org/ViewArticle/aspx?Articleld =7

8. Gaia V, Fry NK, Afshar B, Lu PC, Etienne J, Peduzzi R, et al. Consensus Sequence-Based Scheme for Epidemiological Typing of Clinical and Environmental Isolates of Legionella pneumophila. J Clin Microbiol. 2005;43(5):2047-52.

9. Ratzow S, Gaia V, Helbig JH, Fry NK, Lück PC. Addition of neuA, the gene encoding $\mathrm{N}$-acylneuraminate cytidylyl transferase, increases the discriminatory ability of the consensus sequence-based scheme for typing Legionella pneumophila serogroup 1 strains. J Clin Microbiol. 2007;45(6):1965-8.

10. $2008 / 426 /$ EC: Commission Decision of 28 April 2008 amending Decision 2002/253/EC laying down case definitions for reporting communicable diseases to the Community network under Decision No 2119/98/EC of the European Parliament and of the Council. OJ L. 18 Jun 2008;159:46-90 L 159/65. Available from: http://eur-lex.europa.eu/LexUriServ/LexUriServ.do?uri= OJ:L:2008:159:0046:0090:EN:PDF

11. Lambert M, Moss CW. Cellular fatty acid compositions and isoprenoid quinone contents of 23 Legionella species. J Clin Microbiol. 1989;27(3):465-73.

12. Microbial ID Inc. Microbial identification system operation manual. Newark, Delaware: Microbial ID Inc.; 2002.

13. Mentasti M, Fry NK. European Working Group for Legionella Infections Sequence-Based Typing (SBT) protocol for epidemiological typing of Legionella pneumophila. Version 4.2. Oct 2009; pp 1-9. Available from: http://www.hpabioinformatics.org.uk/legionella/legionella_sbt/php/SBT\%20 protocol\%2ofor\%20website\%202008\%20V4.2.pdf

14. Hunter PR, Gaston MA. Numerical Index of the Discriminatory Ability of Typing Systems: an Application of Simpson' s Index of Diversity. J Clin Microbiol. 1988;26(11):2465-6.

15. Harrison TG, Doshi N, Fry NK, Joseph CA. Comparison of clinical and environmental isolates of Legionella pneumophila obtained in the UK over 19 years. Clin Microbiol Infect. 2007;13(1):78-85.

16. Ricketts K, Joseph C. Legionnaires' disease in Europe: 2003-2004. Euro Surveill. 2005;10(12): pii=588. Available from: http://www.eurosurveillance.org/ViewArticle. aspx?Articleld $=588$

17. Ricketts KD, Joseph CA. Legionnaires' disease in Europe: 2005-2006. Euro Surveill. 2007;12(12): pii=753. Available from: http://www.eurosurveillance.org/ViewArticle. aspx?Articleld $=753$

18. European Centre for Disease Prevention and Control (ECDC). Legionnaires' disease in Europe 2009. Stockholm: ECDC;2011. Available from: http://ecdc.europa.eu/en/publications/ Publications/1109_SR_Legionnaires\%27\%20disease_ Europe_2009.pdf

19. European Centre for Disease Prevention and Control (ECDC). Legionnaires disease in Europe, 2010. Stockholm:ECDC;2012. Available from: http://ecdc.europa.eu/en/publications/ Publications/SUR-Legionnaires-disease-surveillance-2010.pdf

20. Amemura-Maekawa J, Kura F, Helbig JH, Chang B, Kaneko A, Watanabe Y, et al. Characterization of Legionella pneumophila isolates from patients in Japan according to serogroups, monoclonal antibody subgroups and sequence types. J Med Microbiol. 2010;59(Pt 6):653-9.
21. Kozak NA, Benson RF, Brown E, Alexander NT, Taylor TH, Shelton BG, et al. Distribution of lag-1 alleles and sequencebased types among Legionella pneumophila serogroup 1 clinical and environmental isolates in the United States. J Clin Microbiol. 2009;47(8):2525-35.

22. Reimer AR, Au S, Schindle S, Bernard KA. Legionella pneumophila monoclonal antibody subgroups and DNA sequence types isolated in Canada between 1981 and 2009: Laboratory Component of National Surveillance. Eur J Clin Microbiol Infect Dis. 2010;29(2):191-205.

23. Harrison TG, Afshar B, Doshi N, Fry NK, Lee JV. Distribution of Legionella pneumophila serogroups, monoclonal antibody subgroups and DNA sequence types in recent clinical and environmental isolates from England and Wales (2000-2008). Eur J Clin Microbiol Infect Dis. 2009;28(7):781-91.

24. Helbig JH, Bernander S, Castellani Pastoris M, Etienne J, Gaia V, Lauwers S, et al. Pan-European study on culture-proven Legionnaires' disease: distribution of Legionella pneumophila serogroups and monoclonal subgroups. Eur J Clin Microbiol Infect Dis 2002;21(10):710-6.

25. Borchardt J, Helbig JH, Lück PC. Occurrence and distribution of sequence types among Legionella pneumophila strains isolated from patients in Germany: common features and differences to other regions of the world. Eur J Clin Microbiol Infect Dis. 2008;27(1):29-36.

26. Ginevra C, Forey F, Campèse C, Reyrolle M, Che D, Etienne J, et al. Lorraine strain of Legionella pneumophila serogroup 1 , France. Emerg Infect Dis. 2008;14(4):673-5.

27. Den Boer JW, Bruin JP, Verhoef LPB, Van der Zwaluw K, Jansen R, Yzerman EPF. Genotypic comparison of clinical Legionella isolates and patient-related environmental isolates in The Netherlands, 2002-2006. Clin Microbiol Infect. 2008;14(5):459-66.

28. Cazalet C, Jarraud S, Ghavi-Helm Y, Kunst F, Glaser P, Etienne J, et al. Multigenome analysis identifies a worldwide distributed epidemic Legionella pneumophila clone that emerged within a highly diverse species. Genome Res. 2008;18(3):431-41.

29. Tijet N. New Endemic Legionella pneumophila Serogroup I Clones, Ontario, Canada. Emerg Infect Dis. 2010;16(3):447-54. 